\title{
Development of Quinoline-Based Theranostic Ligands for the Targeting of Fibroblast Activation Protein
}

\author{
Thomas Lindner*1, Anastasia Loktev*1-3, Annette Altmann ${ }^{1,2}$, Frederik Giesel ${ }^{1}$, Clemens Kratochwil ${ }^{1}$, Jürgen Debus ${ }^{4,5}$, \\ Dirk Jäger ${ }^{6}$, Walter Mier ${ }^{1}$, and Uwe Haberkorn ${ }^{1,2}$ \\ ${ }^{I}$ Department of Nuclear Medicine, University Hospital Heidelberg, Heidelberg, Germany; ${ }^{2}$ Clinical Cooperation Unit Nuclear \\ Medicine, German Cancer Research Center (DKFZ), Heidelberg, Germany; ${ }^{3}$ Faculty of Biosciences, Heidelberg University, \\ Heidelberg, Germany; ${ }^{4}$ Department of Radiation Oncology, University Hospital Heidelberg, Heidelberg, Germany; ${ }^{5}$ Clinical \\ Cooperation Unit Radiation Oncology, German Cancer Research Center (DKFZ), Heidelberg, Germany; and ${ }^{6}$ Department of \\ Medical Oncology, National Center for Tumor Diseases (NCT), Heidelberg, Germany
}

\section{See an invited perspective on this article on page 1412.}

Fibroblast activation protein (FAP) is overexpressed in cancerassociated fibroblasts and is involved in a variety of tumor-promoting activities such as matrix remodeling, angiogenesis, chemotherapy resistance, and immunosuppression. Because FAP shows low expression in most normal organs, it presents an interesting target for imaging and endoradiotherapy. In this investigation, FAP inhibitors (FAPIs) were modified and optimized for use as theranostic tracers. Methods: FAPIs based on a quinoline structure were synthesized and characterized with respect to binding, internalization, and efflux in cells expressing human and murine FAP as well as CD26. Preclinical pharmacokinetics were determined in tumor-bearing animals with biodistribution experiments and smallanimal PET. Finally, a proof-of-concept approach toward imaging and therapy was chosen for 2 patients with metastasized breast cancer. Results: Of 15 synthesized FAPIs, FAPI-04 was identified as the most promising tracer for clinical application. Compared with the previously published ligand, FAPI-02, FAPI-04 showed excellent stability in human serum, higher affinity for FAP as opposed to CD26, and slower excretion in vitro. In vivo, a higher SUV was reached in tumor-bearing animals, leading to larger areas under the curve as calculated from biodistribution experiments. Finally, PET/CT scans with ${ }^{68} \mathrm{Ga}-\mathrm{FAPI}-04$ in 2 patients with metastasized breast cancer revealed high tracer uptake in metastases and a reduction in pain symptoms after therapy with a considerably low dose of ${ }^{90}$ Y-FAPI-04. Conclusion: FAPI-04 represents a promising tracer for both diagnostic imaging and, possibly, targeted therapy of malignant tumors with a high content of activated fibroblasts, such as breast cancer.

Key Words: oncology; PET; radiopharmaceuticals; FAP; small molecules; theranostic

J Nucl Med 2018; 59:1415-1422

DOI: 10.2967/jnumed.118.210443

Received Feb. 23, 2018; revision accepted Mar. 22, 2018.

For correspondence or reprints contact: Uwe Haberkorn, Department of Nuclear Medicine, University Hospital Heidelberg, Im Neuenheimer Feld 400, 69120 Heidelberg, Germany.

E-mail: uwe.haberkorn@med.uni-heidelberg.de

${ }^{*}$ Contributed equally to this work.

Published online Apr. 6, 2018.

COPYRIGHT (C 2018 by the Society of Nuclear Medicine and Molecular Imaging.

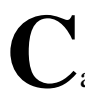

ancer-associated fibroblasts modulate the microenvironment of malignant tumors by secreting factors that regulate both malignant cells and nonmalignant cells such as immune and endothelial cells (1). Although heterogeneous in their origin, cancer-associated fibroblasts have common properties distinct from normal fibroblasts and show expression of proteins not found in their normal counterparts. One of these proteins is the fibroblast activation protein (FAP), a type II transmembrane serine protease with both dipeptidyl peptidase activity and endopeptidase activity (2-4).

FAP expression is associated with a poor prognosis in a variety of tumors, such as colon (5), pancreatic (6), ovarian (7), and hepatocellular carcinoma (8). It plays multiple biologic roles in cancer. First, via its peptidase activity, FAP leads to matrix digestion and remodeling of the tumor microenvironment, enabling invasion and migration of tumor cells (9). Because neuropeptide $\mathrm{Y}$ is a natural substrate of FAP and the cleavage product has been shown to be proangiogenic, FAP is considered to be involved in tumor angiogenesis, as has been substantiated by studies showing a correlation between FAP expression and microvessel density in tumors $(10,11)$.

Furthermore, besides its enzyme function, FAP activates cell signaling by forming complexes with other proteins-for example, $\beta 1$ integrins such as $\alpha 3 \beta 1(12,13)$.

Because cancer-associated fibroblasts are the primary source of collagen I, which contributes to decreased chemotherapeutic drug uptake and thereby plays a role in regulating the sensitivity of tumors toward chemotherapy, elimination of these fibroblasts via targeting of FAP may lead to increased uptake of chemotherapeutic drugs and greater therapeutic efficiency (14). An immunosuppressive role has also been described (15).

In addition, FAP has other favorable properties qualifying it as a promising target for diagnosis and therapeutic intervention: besides expression in some nonmalignant conditions such as wound healing, rheumatoid arthritis, atherosclerotic plaques, and diseases leading to fibrosis, FAP is expressed in more than $90 \%$ of human epithelial cancers, is absent from normal tissues in adult humans, and has a large extracellular domain, with the catalytic site also located extracellularly. These properties ensure a low background activity with high image contrast and a low frequency of side effects for FAP-targeting molecules, the possibility of application in many different tumor entities, and the design of 
combination therapies targeting both tumor cells and the stromal components of a tumor. Such targeting of the stromal cells has been done using a variety of molecules, including antibodies (16$18)$, chimeric antigen receptor $\mathrm{T}$ cells $(19,20)$, immunoconjugates (21), peptide-drug complexes bearing the consensus sequence for the enzymatic activity $(22,23)$, vaccines $(14,24)$, and small molecules for the inhibition of FAP enzyme activity (25). Another approach is the use of these small molecules as carriers of radioactivity into the tumor for both imaging and therapy. In a previous paper, we showed that a FAP inhibitor (FAPI), FAPI-02, rapidly accumulates in FAP-expressing cells, tumor xenografts, and patients (26). Although high image contrast was obtained, the tumor retention time was relatively short, a quality that presents no problem for diagnosis but may need improvement for therapy. In this investigation, we designed variants of FAPI-02 with the goal of increasing tumor retention time and thereby developing a theranostic FAP-targeting agent.

\section{MATERIALS AND METHODS}

\section{Synthesis and Radiolabeling}

As an example of the synthetic pathway toward the FAPI derivatives, the pathway for FAPI-04 is given in Supplemental Figure 1 (supplemental materials are available at http://jnm.snmjournals.org). A detailed compilation of the precursors, the intermediates, and their syntheses can be found in the supplemental material. ${ }^{177} \mathrm{Lu}$ and ${ }^{68} \mathrm{Ga}$ were chelated after $\mathrm{pH}$ adjustment with sodium acetate. The reaction mixture was heated to $95^{\circ} \mathrm{C}$ for $10 \mathrm{~min}$, and the completeness of the reaction was checked by radio-liquid chromatography. The ${ }^{68} \mathrm{Ga}$ compounds were processed by solid-phase extraction before PET. Stability in human serum was determined by precipitation of samples and radiochromatographic analysis of the supernatant. The stability of the compounds was checked for FAPI-04, as an example, by incubation in human serum at $37^{\circ} \mathrm{C}$ (Supplemental Fig. 2).

\section{Cell Culture}

HT-1080 cells transfected with the human or murine FAP gene, as well as CD26-transfected human embryonic kidney cells (obtained from Stefan Bauer, NCT, Heidelberg, Germany (27)), were cultivated in Dulbecco modified Eagle medium containing 10\% fetal calf serum at $37^{\circ} \mathrm{C}$ in $5 \%$ carbon dioxide.

For radioligand binding studies, cells were seeded in 6-well plates and cultivated for $48 \mathrm{~h}$ to a final confluence of approximately $80 \%-$ $90 \%\left(1.2-2 \times 10^{6}\right.$ cells per well). The medium was replaced by $1 \mathrm{~mL}$ of fresh medium without fetal calf serum. The radiolabeled compound $\left({ }^{177} \mathrm{Lu}\right.$-labeled FAPIs; specific activity, $\left.200 \mathrm{nmol} / \mathrm{GBq}\right)$ was added to the cell culture and incubated for different intervals ranging from $10 \mathrm{~min}$ to $24 \mathrm{~h}$. Competition experiments were performed by simultaneous exposure to unlabeled $\left(10^{-5}\right.$ to $\left.10^{-9} \mathrm{M}\right)$ and radiolabeled compound for $60 \mathrm{~min}$. In all experiments, the cells were washed twice with $1 \mathrm{~mL}$ of phosphate-buffered saline, $\mathrm{pH} 7.4$, and subsequently lysed with $1.4 \mathrm{~mL}$ of lysis buffer $(0.3 \mathrm{M} \mathrm{NaOH}, 0.2 \%$ sodium dodecyl sulfate). Radioactivity was determined in a $\gamma$-counter (Cobra II; Packard), normalized to $10^{6}$ cells, and calculated as percentage injected dose (\%ID). Cell efflux was determined after incubation of the cells with the tracer for $60 \mathrm{~min}$. Afterward, the radioactive medium was removed, and the cells were washed and incubated in nonradioactive medium for 1, 2, 4, or $24 \mathrm{~h}$. After 2 washings, the cells were lysed and the radioactivity counted. Each experiment was performed 3 times, and 3 repetitions per independent experiment were acquired.

For internalization experiments, the cells were incubated with the radiolabeled compound for $60 \mathrm{~min}$ at $37^{\circ} \mathrm{C}$ and $4^{\circ} \mathrm{C}$. Cellular uptake was terminated by removing medium from the cells and washing twice with $1 \mathrm{~mL}$ of phosphate-buffered saline. Subsequently, the cells were incubated with $1 \mathrm{~mL}$ of glycine- $\mathrm{HCl}(1 \mathrm{M}, \mathrm{pH} 2.2)$ for $10 \mathrm{~min}$ at room temperature to remove the surface-bound activity. The cells were washed with $2 \mathrm{~mL}$ of ice-cold phosphate-buffered saline and lysed with $1.4 \mathrm{~mL}$ of lysis buffer to determine the internalized fraction. For the cells incubated at $4^{\circ} \mathrm{C}$, all washing and elution steps were performed using ice-cold buffers. The radioactivity was measured using a $\gamma$-counter, normalized to 1 million cells, and calculated as \%ID.

\section{Animal Studies}

For in vivo experiments, 8-wk-old BALB/c nu/nu mice (Charles River) were subcutaneously inoculated into the right trunk with $5 \times$ $10^{6}$ HT-1080 FAP cells. When the tumor had grown to approximately $1 \mathrm{~cm}^{3}$, the radiolabeled compound $\left({ }^{177} \mathrm{Lu}\right.$-labeled FAPIs; specific activity, $200 \mathrm{nmol} / \mathrm{GBq}$ ) was injected via the tail vein. For organ distribution, the animals ( $n=3$ for each time point) were sacrificed after the indicated time points (from $30 \mathrm{~min}$ to $24 \mathrm{~h}$ ). The distributed radioactivity was measured in all dissected organs and in blood using a $\gamma$-counter (Cobra Autogamma; Packard). The values are expressed as \%ID per gram of tissue (\%ID/g). PET imaging was performed using a small-animal PET scanner (Inveon; Siemens). The mice were injected with ${ }^{68} \mathrm{Ga}-F A P I-02(80 \mathrm{nmol} / \mathrm{GBq})$. Within the first $60 \mathrm{~min}$, a dynamic scan was performed, followed by a static scan from 120 to $140 \mathrm{~min}$ after injection. Images were reconstructed iteratively using the 3-dimensional maximum a priori ordered-subset expectation maximization (Siemens) and were converted to SUV images. Quantification was done using a region-of-interest technique and expressed as SUV. Imaging was also done in animals bearing SK-LMS-1 tumors, a model in which the tumor cells are FAP-negative and only activated mouse fibroblasts are present. A blocking experiment was performed by adding $30 \mathrm{nmol}$ of unlabeled precursor to the solution of ${ }^{68} \mathrm{Ga}$ FAPI-04 before injection. All animal experiments were conducted in compliance with the German animal protection laws.

\section{Clinical PET/CT Imaging}

Diagnostic imaging of 2 patients with ${ }^{68} \mathrm{Ga}$-FAPI-04 PET/CT for medical reasons was performed under the conditions of the updated declaration of Helsinki (section 37, unproven interventions in clinical practice) and in accordance with the German Pharmaceuticals Law (section 13, 2b). The tracer was injected intravenously $(80 \mathrm{nmol} /$ $\mathrm{GBq}$ ), and images were obtained $10 \mathrm{~min}, 1 \mathrm{~h}$, and $3 \mathrm{~h}$ later. The images were obtained on a Biograph mCT Flow PET/CT scanner (Siemens Medical Solutions) using the following parameters: a 5-mm slice thickness, an increment of 3-4 mm, a soft-tissue reconstruction kernel, and CARE Dose4D (Siemens Medical Solutions). Immediately after the CT component had been acquired, whole-body PET was performed in 3 dimensions (matrix, $200 \times 200$ ) in FlowMotion (Siemens Medical Solutions) at a rate of $0.7 \mathrm{~cm} / \mathrm{min}$. The emission data were corrected for random, scatter, and decay events. Images were reconstructed using ordered-subset expectation maximization with 2 iterations and 21 subsets and were Gauss-filtered to a transaxial resolution of $5 \mathrm{~mm}$ in full width at half maximum. Attenuation was corrected using the low-dose nonenhanced CT data. SUVs were quantitatively assessed using a region-of-interest technique. All patients gave written informed consent. The evaluation was approved by our institutional ethical review board (approval S-016/2018).

\section{Therapeutic Application}

One patient with metastasized breast cancer was treated with 2.9 GBq of ${ }^{90}$ Y-FAPI-04 (24 nmol/GBq). FAPI-04 was labeled with a ${ }^{90}$ Y-chloride solution (Yttriga; Eckert and Ziegler Radiopharma $\mathrm{GmbH}$ ) and injected intravenously via a low-protein-binding sterile filter system (Filtropur S 0.2; Sarstedt). $\gamma$-camera images of the bremsstrahlung were acquired $3 \mathrm{~h}$ and $1 \mathrm{~d}$ after tracer administration using a Hawkeye SPECT/CT system (GE Healthcare). 


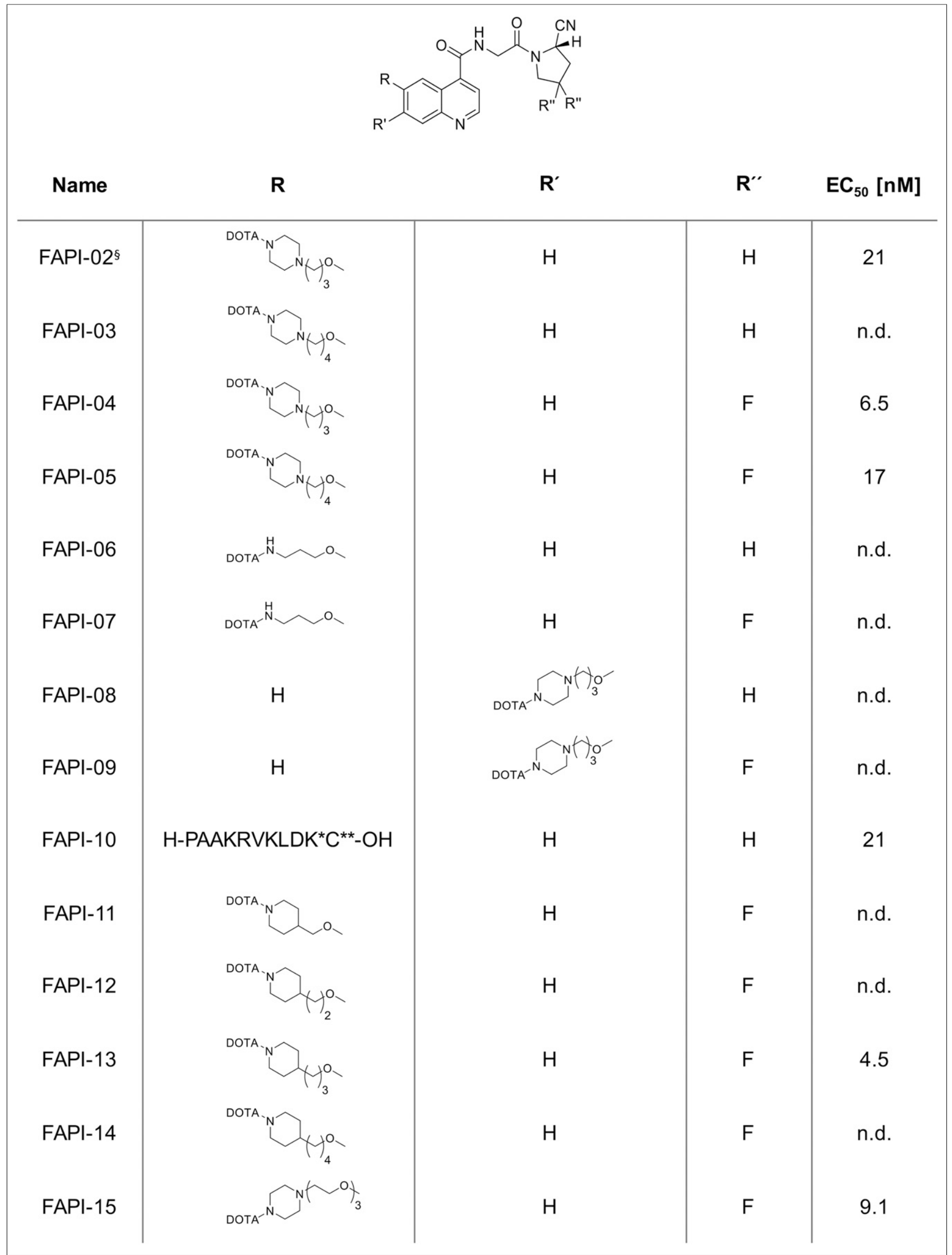

FIGURE 1. Overview of synthesized FAPI derivatives. $E_{50}$ obtained by competition experiments is shown for selected compounds. ${ }^{\S}$ Discussed in previous publication (28). ${ }^{*} \varepsilon$-amine modified by DOTA. ${ }^{\star \star}$ Cysteine-thiol attached to maleimide-carrying piperazinylpropoxy moiety. n.d. $=$ not determined. 


\section{RESULTS}

\section{Synthesis and Radiolabeling}

Following the synthetic pathway shown in Supplemental Figure 1,11 new compounds and a small-molecule-peptide conjugate were synthesized. An overview of the synthesized compounds is given in Figure 1. To trace the peptide via radioactive labeling, $\varepsilon$-DOTA-lysine was inserted C-terminally between the end of the NLS sequence and the conjugated cysteine residue.

\section{Cell-Based Experiments}

To evaluate the binding and internalization properties of the FAPI derivatives, radioligand binding assays were performed using FAPexpressing HT-1080 cells (Fig. 2). All tracers showed an almost complete internalization, with values over $90 \%$. Because of insufficient binding to human FAP after $24 \mathrm{~h}$, the compounds FAPI-06, FAPI-07, FAPI-08, and FAPI-09 were not characterized further. Although they showed acceptable accumulation after $24 \mathrm{~h}$, the evaluation of FAPI-11 and FAPI-12 was not pursued because of bad performance after 1 and $4 \mathrm{~h}$ of incubation. To verify the target specificity, binding assays were also performed using human embryonic kidney cells expressing murine FAP and dipeptidyl peptidase 4 (CD26), which show a high homology to human FAP (Supplemental Fig. 2). In these experiments, FAPI-02 and FAPI-04 showed a strong binding to murine FAP, with significantly higher values for FAPI-04, and no binding to CD26. However, FAPI-15 also had a low affinity to $\mathrm{CD} 26$. Calculation of the ratio of murine FAP binding to CD26 binding revealed values of 45, 750, and 38 for FAPI-02, FAPI-04, and FAPI-15, respectively (Supplemental Fig. 2).

In the first set of experiments, the difluoroproline analogs FAPI04 and FAPI-05 were analyzed to investigate the influence of the presence of fluorine and the difference between a propyl linkage and a butyl linkage of the piperazine and quinoline components of the radiotracers. For this purpose, the half-maximal effective concentration $\left(\mathrm{EC}_{50}\right)$ for each FAPI was determined by a competition assay, which demonstrated a higher target specificity for FAPI-04 (6.5 nM) than for FAPI-05 (17.2 nM; Supplemental Fig. 3). Efflux experiments showed that FAPI-04 and FAPI-05 were excreted considerably more slowly than FAPI-02, resulting in increased half-lives of 3.0 and $2.8 \mathrm{~h}$ (FAPI-02, $1.7 \mathrm{~h}$; Supplemental Fig. 4). FAPI-13 was characterized in more detail because of its resemblance to the compound FAPI-04 and showed the lowest $\mathrm{EC}_{50}$ in this set of data (4.5 nM; Supplemental Fig. 3).

The compounds FAPI-10 and FAPI-15 were examined because of their unique modifications: FAPI-10 is a peptide conjugate of the FAPI-02 core with the intention of localizing the radioactivity in the nucleus, and FAPI-15 is similar to FAPI-04 but possesses a triethylene glycol spacer instead of the 1,3-propyl moiety. In both cases, $\mathrm{EC}_{50}$ was comparable to that of the parenting radiotracer.

\section{Organ Distribution}

To evaluate distribution, tumor uptake, and excretion of selected FAPI derivatives, biodistribution studies were performed on mice bearing human HT-1080 FAP-expressing xenografts. Figure 3 shows the results of the biodistribution assays performed with FAPI-04 and FAPI-13 compared with the existing data for FAPI02. FAPI-10 was discarded because of a relatively low tumor accumulation at 1 and $4 \mathrm{~h}$ after administration. Moreover, it accumulated strongly within the kidneys $(\leq 10 \%$ ID/g; FAPI-02, 1.6 $\% \mathrm{ID} / \mathrm{g}$ ), with high kidney values even after $24 \mathrm{~h}$ (Supplemental Fig. 5). FAPI-05 produced results comparable to FAPI-02 but was discarded with regard to the better-performing derivatives FAPI-

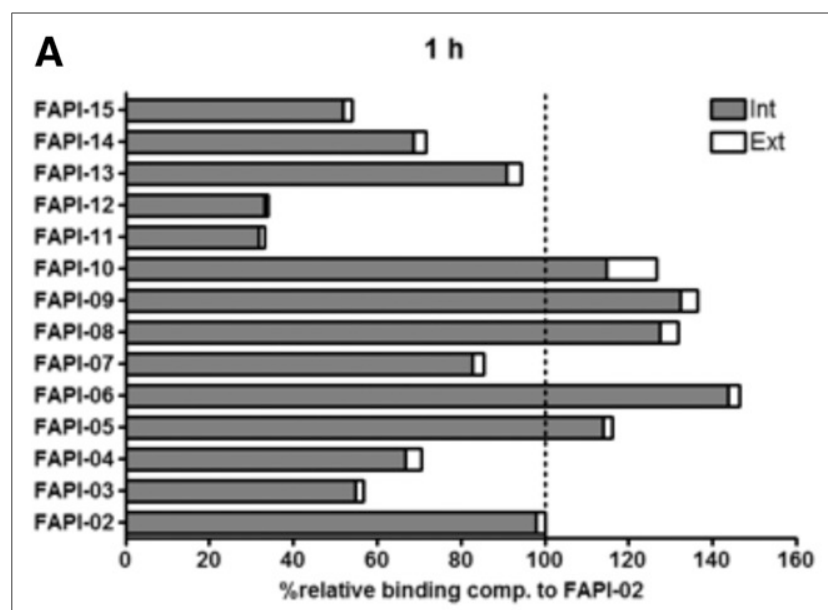

B

$4 \mathrm{~h}$
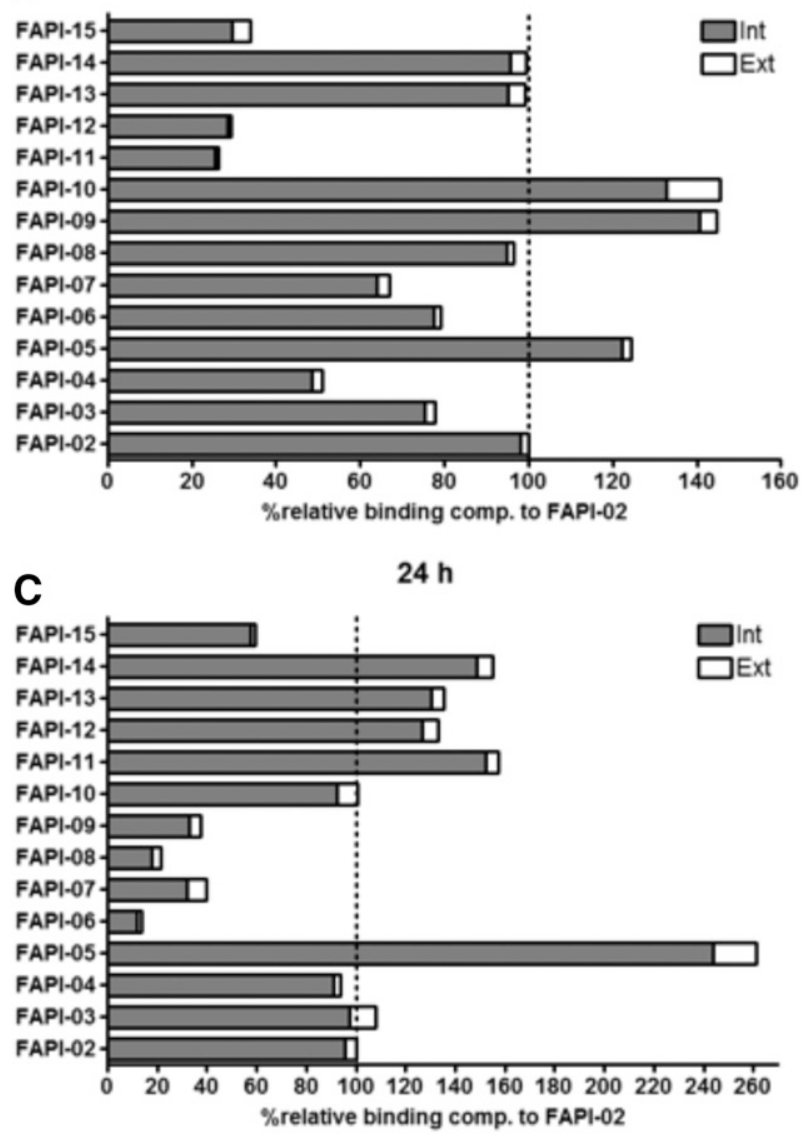

FIGURE 2. Relative binding and internalization rates of ${ }^{177} \mathrm{Lu}$-labeled FAPI derivatives compared with FAPI-02 (set to 100\%) on FAP-expressing HT1080 cells at $1 \mathrm{~h}(\mathrm{~A}), 4 \mathrm{~h}(\mathrm{~B})$, and $24 \mathrm{~h}(\mathrm{C})$ after radiotracer administration.

04 and FAPI-13. Tracer uptake in most normal tissues was slightly higher for FAPI-04 than for FAPI-02 and was further increased for FAPI-13. Tumor accumulation was higher for FAPI-04 and FAPI13 than for FAPI-02, especially after $24 \mathrm{~h}$. A calculation of the area the under curve is shown in Supplemental Table 1. The values for FAPI-02, FAPI-04, and FAPI-15 were 64, 99.4, and 157.5, respectively. However, the tumor-to-blood ratio favored FAPI-04 over FAPI-13 (Supplemental Fig. 6). 


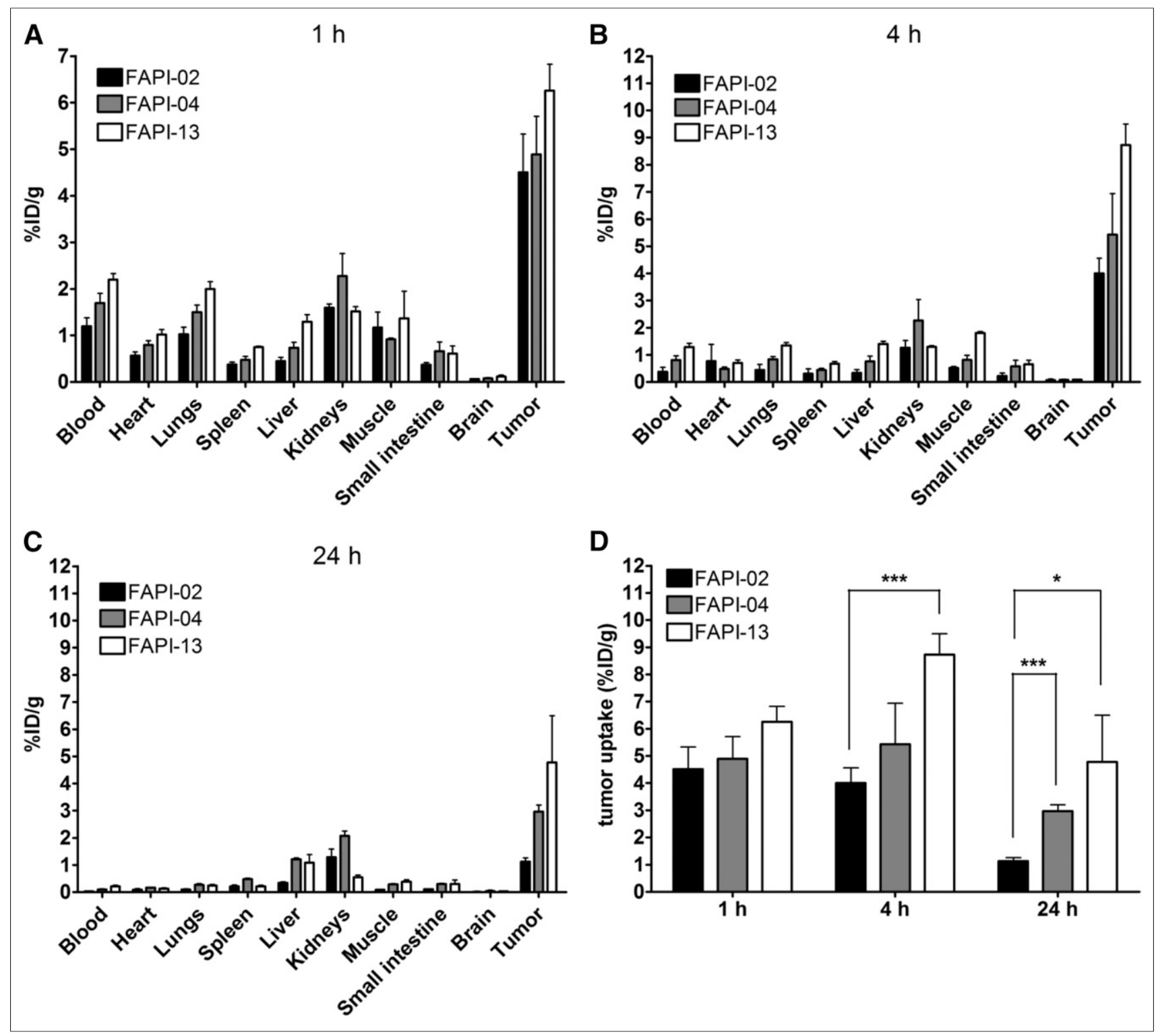

FIGURE 3. (A-C) Biodistribution of selected FAPI derivatives in HT-1080 FAP xenografts at $1 \mathrm{~h}(\mathrm{~A}), 4 \mathrm{~h}(\mathrm{~B})$, and $24 \mathrm{~h}(\mathrm{C})$ after radiotracer administration. (D) Tumor uptake of selected compounds.

\section{Small-Animal PET Imaging}

For further characterization of the remaining FAPIs, smallanimal PET studies were conducted with HT-1080 FAP-xenografted mice (Figs. 4 and 5). ${ }^{68}$ Ga-FAPI-02 and ${ }^{68}$ Ga-FAPI-04 demonstrated the highest tumor uptake at $1 \mathrm{~h}$ after injection $\left(\mathrm{SUV}_{\max }\right.$, 0.88 and 1.2, respectively), with no significant decrease within $2 \mathrm{~h}$ (SUV, 0.71 and 1.1, respectively). In contrast, tumor accumulation of ${ }^{68} \mathrm{Ga}$-FAPI-13 increased steadily for up to $2 \mathrm{~h}$ after injection (SUV at $1 \mathrm{~h}, 0.93$; at $2 \mathrm{~h}, 0.97$ ). However, uptake was higher in background, spinal cord, and bone (Supplemental Fig. 7). Additionally, the applicability of ${ }^{68} \mathrm{Ga}$-FAPI-04 in a second xenograft model, SK-LMS-1 was successfully demonstrated (Fig. 4). Furthermore, target specificity was elucidated by a blocking experiment on an HT-1080 FAP xenograft. As shown in Figure 5, accumulation in the tumor was suppressed effectively by coadministration of unlabeled compound.

\section{Clinical Application in Patients with Breast Cancer}

Diagnostic PET/CT scans were performed $10 \mathrm{~min}, 1 \mathrm{~h}$, and $3 \mathrm{~h}$ after intravenous administration of ${ }^{68} \mathrm{Ga}-\mathrm{FAPI}-04$ in 2 patients with metastasized breast cancer (Figs. 6 and 7). In both patients, accumulation of the tracer in metastases was robust $\left(\mathrm{SUV}_{\max }, 7-\right.$ 15.5 for patient in Fig. 6 and 15.3-29.9 for patient in Fig. 7). In contrast, tracer uptake in normal tissue was very low (Supplemental Table 2). The radioactivity cleared rapidly from the bloodstream and was excreted predominantly via the kidneys, resulting in high-contrast images. In addition to imaging, therapy with $2.9 \mathrm{GBq}$ of ${ }^{90}$ Y-FAPI-04 was used in one patient. The bremsstrahlung images showed accumulation of the tracer at $3 \mathrm{~h}$ and even at $1 \mathrm{~d}$ after injection in this patient. This rather low dose led to a significant reduction of pain medication in this patient (from baseline morphine and 3-4 additional morphine administrations per day to baseline medication only). 


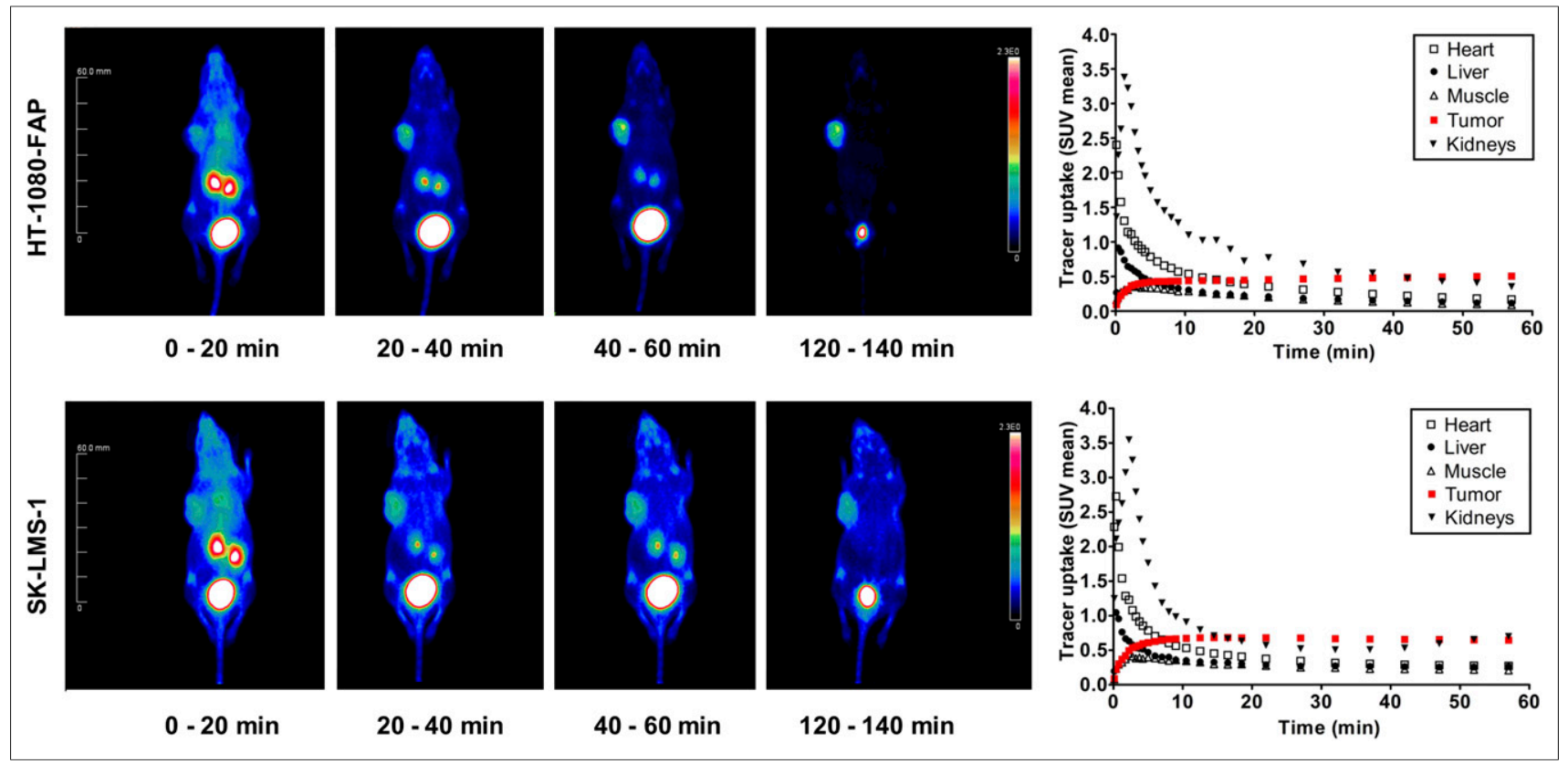

FIGURE 4. PET imaging of FAPI-04 in mice bearing SK-LMS-1 and HT-1080 FAP tumors, along with corresponding time-activity curves.

\section{DISCUSSION}

In this work, several approaches were investigated to improve the pharmacokinetic properties of the quinoline-based radiopharmaceuticals developed by our laboratory (26). Herein, particular focus was on the elaboration of a theranostic tracer (a tracer that can be used for both diagnostics and therapy). This concept is based on the linking of the FAP-targeting moiety to the chelator DOTA, which allows the incorporation of different isotopes suitable for imaging or therapeutic purposes. Using the ligand FAPI-04, we were able to demonstrate the targeted delivery of the positronemitting ${ }^{68} \mathrm{Ga}$ as well as the therapeutic nuclide ${ }^{90} \mathrm{Y}$ in a clinical setting. The main challenge for potential therapeutic application of the tracer was in optimizing its tumor uptake and retention time. We addressed this task by assessing a series of novel compounds based on the initial FAP inhibitor, FAPI-02. The most striking improvement was in the use of 4,4-difluoroproline, which has already been successful with respect to enzyme inhibition as described by Jansen et al. (28). By this substitution, the $\mathrm{EC}_{50}$ of ${ }^{177} \mathrm{Lu}$-FAPI-04 was reduced by a factor of 3, compared with ${ }^{177} \mathrm{Lu}-\mathrm{FAPI}-02$. Also, the difference in affinity between FAP and CD26 was shifted in favor of ${ }^{177}$ Lu-FAPI-04 as shown by ${ }^{177} \mathrm{Lu}-\mathrm{FAP} / \mathrm{CD} 26$ binding ratios of 45 and 750 for FAPI-02 and ${ }^{177} \mathrm{Lu}-\mathrm{FAPI}-04$, respectively. In addition, the efflux experiments showed a significantly slower washout for ${ }^{177}$ Lu-FAPI-04 than for ${ }^{177}$ Lu-FAPI-02, resulting in an increased half-life of $3.0 \mathrm{~h}$ for ${ }^{177} \mathrm{Lu}$-FAPI-04, versus $1.7 \mathrm{~h}$ for ${ }^{177} \mathrm{Lu}-$ FAPI-02 (Supplemental Fig. 4).

Although in vitro ${ }^{177} \mathrm{Lu}$-FAPI-02 uptake was higher than ${ }^{177} \mathrm{Lu}$-FAPI-04 uptake after 1 and $4 \mathrm{~h}$ of incubation and equaled ${ }^{177} \mathrm{Lu}-$ FAPI-04 uptake after $24 \mathrm{~h}$, the FAPI-04 PET imaging and biodistribution studies in small animals demonstrated higher accumulation, longer dwell times, and no significant increase in background activity. Compared with ${ }^{177}$ Lu-FAPI-04, ${ }^{177}$ Lu-FAPI-13 showed higher tumor uptake leading to larger areas under the curve. However, retention of the tracer in the blood was higher, leading to lower tumor-to-blood ratios. This may lead to higher hematotoxicity during treatment,
FIGURE 5. PET imaging of FAPI-04 in HT-1080 FAP tumor-bearing mice with and without simultaneous injection of unlabeled FAPI-04 as competitor, along with corresponding timeactivity curves. 


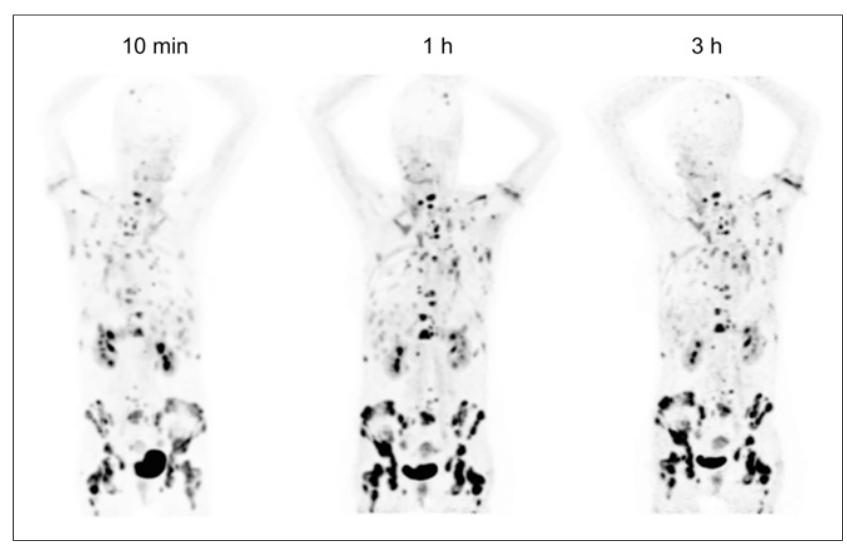

FIGURE 6. PET maximum-intensity projections of patient with metastasized breast cancer $10 \mathrm{~min}, 1 \mathrm{~h}$, and $3 \mathrm{~h}$ after administration of $263 \mathrm{MBq}$ of ${ }^{68} \mathrm{Ga}-\mathrm{FAPI}-04$. Activity is seen in renal pelvis, bladder, and metastases. Normal organs show low uptake resulting in high image contrast.

narrowing the therapeutic window. Because of these improvements, therapeutic implementation of ${ }^{90}$ Y-labeled FAPI-04 was initiated. A previous stability analysis of ${ }^{177} \mathrm{Lu}-\mathrm{FAPI}-04$ in human serum revealed no degradation during $24 \mathrm{~h}$, qualifying this compound for clinical translation (Supplemental Fig. 1).

A structure-activity relationship analysis was done for modifications of the heterocyclic segment and the position of the linker. The simple 3-amino-1-propyl derivatives FAPI-06 and FAPI-07 showed suitable cell binding at 1 and $4 \mathrm{~h}$ but were almost completely eliminated after $24 \mathrm{~h}$, demonstrating that the heterocyclic segment is necessary for sufficient retention in the tumor cells. The 7-quinolyl-linked compounds FAPI-08 and FAPI-09 revealed the same pharmacokinetic profile in the course of incubation, as might be explained by the removal of the radiolabeled moiety. This indicates that the position of the linker at the quinoline moiety is equally essential. In FAPI-06 and FAPI-07, the bond between DOTA and the propylamine is far more accessible than the piperazine-bound DOTA of FAPI-02 and FAPI-04, and an enzymatic breakdown can proceed much more quickly.

The binding to albumin or other plasma proteins with a longer retention time in the circulation is seen as a knockout criterion for

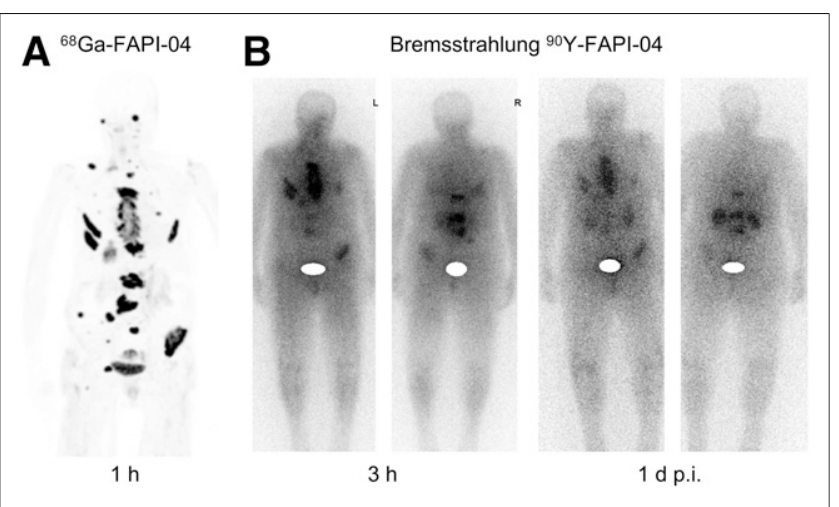

FIGURE 7. (A) PET maximum-intensity projection of patient with metastasized breast cancer $1 \mathrm{~h}$ after administration of $270 \mathrm{MBq}$ of ${ }^{8} \mathrm{Ga}-$ FAPI-04. Robust uptake is seen in metastases. (B) Bremsstrahlung images showing uptake at $3 \mathrm{~h}$ and even $1 \mathrm{~d}$ after treatment with ${ }^{90} \mathrm{Y}$ FAPI-04 in same patient. possible theranostics and the reason ${ }^{177} \mathrm{Lu}-\mathrm{FAPI}-13$ was not considered for further development despite having the lowest $\mathrm{EC}_{50}$ and the highest tumor accumulation of the compared compounds in tumor-bearing animals. The higher lipophilicity of the piperidine moiety is presumably the cause for the higher affinity, better tissue permeation, and longer target prevalence but leads to unspecific binding to serum proteins, thus competing with the attachment to FAP and having a negative impact on the pharmacokinetic properties $(29,30)$.

The in vitro and in vivo results led to the transfer of FAPI-04 to clinical diagnosis and therapy in 2 patients with metastasized breast cancer. As seen in the animal experiments, PET/CT imaging in these patients showed a rapid, predominantly renal washout from the body and an equally rapid accumulation in tumors (SUV, 7-29.9), leading to visualization of metastases even at $10 \mathrm{~min}$ after tracer administration. Besides this finding, radioactivity was seen only in the renal pelvis and the bladder, with no accumulation in the renal parenchyma or any other organ, making FAPI-04 an attractive tracer for both diagnostic and therapeutic applications. To fit the physical half-life of the isotope used for therapy to the retention in the tumor, ${ }^{90} \mathrm{Y}$, with a half-life of $64 \mathrm{~h}$, was chosen for treatment. In a proof-of-principle approach, a patient was treated with $2.9 \mathrm{GBq}$ of ${ }^{90}$ Y-FAPI-04, which resulted in visualization of the metastases in bremsstrahlung images at $24 \mathrm{~h}$ after tracer administration (Fig. 7). Clinically, this treatment was associated with a significant reduction in pain medication. Furthermore, no side effects were observed, especially with respect to hematotoxicity. Because tracer uptake was low in all normal organs, we expect that the dose can be significantly increased to obtain tumoricidal effects. However, any such dose increase in future approaches has to be based on dosimetric calculations and dose escalation regimens.

\section{CONCLUSION}

On the basis of our previous work on quinoline-based FAPtargeted radiopharmaceuticals, we successfully developed FAPI-04 as a theranostic tool. The pharmacokinetic profile of the compound was improved by incorporation of the 4,4-difluoroprolyl building block. Moreover, a comprehensive analysis of the synthesized compounds provided valuable insight into the structure-activity relationship of the chelator-carrying side chain.

Of all the derivatives tested, FAPI-04 is the most suitable for potential application as a theranostic tracer. Similar to its precursor, FAPI-02, FAPI-04 shows rapid internalization into FAP-positive tumors and fast clearance from the body, resulting in very fast accumulation at tumor sites (10 min after tracer administration) and comparable tumor-to-organ ratios. Moreover, the effective tumor uptake after $24 \mathrm{~h}-100 \%$ higher for FAPI-04 than for FAPI-02 - is of great benefit regarding theranostic application of the tracer. A proofof-concept approach that was applied to 2 patients gave excellent performance results for diagnosis and promising first results for therapy. Future development will concentrate on the design of compounds for labeling with isotopes that have physical characteristics different from ${ }^{68} \mathrm{Ga}$ and ${ }^{90} \mathrm{Y}$, such as ${ }^{188} \mathrm{Re},{ }^{64} \mathrm{Cu}$, or ${ }^{212} \mathrm{~Pb}$.

\section{DISCLOSURE}

Uwe Haberkorn, Anastasia Loktev, Thomas Lindner, and Walter Mier have been named in a patent application (EP 18155420.5) for quinolone-based FAP-targeting agents for 
imaging and therapy in nuclear medicine. No other potential conflict of interest relevant to this article was reported.

\section{ACKNOWLEDGMENTS}

We gratefully acknowledge Stefan Bauer (National Center for Tumor Diseases, Heidelberg) for supplying the FAP and CD26transfected cell lines. We thank Christian Kleist, Susanne Krämer, Stephanie Biedenstein, Kirsten Kunze, Irina Kupin, Vanessa Kohl, Marlene Tesch, Iris Morr, Sabine Weiss, Christiane Brenner, Karin Leotta, and Ursula Schierbaum for excellent technical assistance. This work was funded in part by grant 13N 13341 from the Federal Ministry of Education and Research.

\section{REFERENCES}

1. Gascard P, Tlsty TD. Carcinoma-associated fibroblasts: orchestrating the composition of malignancy. Genes Dev. 2016;30:1002-1019.

2. Park JE, Lenter MC, Zimmermann RN, Garin-Chesa P, Old LJ, Rettig WJ. Fibroblast activation protein, a dual specificity serine protease expressed in reactive human tumor stromal fibroblasts. J Biol Chem. 1999;274:36505-36512.

3. Rettig WJ, Garin-Chesa P, Healey JH, et al. Regulation and heteromeric structure of the fibroblast activation protein in normal and transformed cells of mesenchymal and neuroectodermal origin. Cancer Res. 1993;53:3327-3335.

4. Garin-Chesa P, Old LJ, Rettig WJ. Cell surface glycoprotein of reactive stromal fibroblasts as a potential antibody target in human epithelial cancers. Proc Natl Acad Sci USA. 1990;87:7235-7239.

5. Henry LR, Lee HO, Lee JS, et al. Clinical implications of fibroblast activation protein in patients with colon cancer. Clin Cancer Res. 2007;13:1736-1741.

6. Cohen SJ, Alpaugh RK, Palazzo I, et al. Fibroblast activation protein and its relationship to clinical outcome in pancreatic adenocarcinoma. Pancreas. 2008;37: 154-158.

7. Zhang Y, Tang H, Cai J, et al. Ovarian cancer-associated fibroblasts contribute to epithelial ovarian carcinoma metastasis by promoting angiogenesis, lymphangiogenesis and tumor cell invasion. Cancer Lett. 2011;303:47-55.

8. Ju MJ, Qiu SJ, Fan J, et al. Peritumoral activated hepatic stellate cells predict poor clinical outcome in hepatocellular carcinoma after curative resection. Am J Clin Pathol. 2009;131:498-510.

9. Chen WT, Kelly T. Seprase complexes in cellular invasiveness. Cancer Metastasis Rev. 2003;22:259-269.

10. Keane FM, Nadvi NA, Yao TW, Gorrell MD, Neuropeptide Y. B-type natriuretic peptide, substance $\mathrm{P}$ and peptide $\mathrm{YY}$ are novel substrates of fibroblast activation protein-alpha. FEBS J. 2011;278:1316-1332.

11. Huang Y, Wang S, Kelly T. Seprase promotes rapid tumor growth and increased microvessel density in a mouse model of human breast cancer. Cancer Res. 2004; 64:2712-2716.

12. Kelly T. Fibroblast activation protein-alpha and dipeptidyl peptidase IV (CD26): cell-surface proteases that activate cell signaling and are potential targets for cancer therapy. Drug Resist Updat. 2005;8:51-58.
13. Mueller SC, Ghersi G, Akiyama SK, et al. A novel protease-docking function of integrin at invadopodia. J Biol Chem. 1999;274:24947-24952.

14. Loeffler M, Kruger JA, Niethammer AG, Reisfeld RA. Targeting tumor-associated fibroblasts improves cancer chemotherapy by increasing intratumoral drug uptake. J Clin Invest. 2006;116:1955-1962.

15. Kraman M, Bambrough PJ, Arnold JN, et al. Suppression of antitumor immunity by stromal cells expressing fibroblast activation protein-alpha. Science. 2010; 330:827-830.

16. Welt S, Divgi CR, Scott AM, et al. Antibody targeting in metastatic colon cancer: a phase I study of monoclonal antibody F19 against a cell-surface protein of reactive tumor stromal fibroblasts. J Clin Oncol. 1994;12:1193-1203.

17. Scott AM, Wiseman G, Welt S, et al. A phase I dose-escalation study of sibrotuzumab in patients with advanced or metastatic fibroblast activation proteinpositive cancer. Clin Cancer Res. 2003;9:1639-1647.

18. Hofheinz RD, al-Batran SE, Hartmann F, et al. Stromal antigen targeting by a humanised monoclonal antibody: an early phase II trial of sibrotuzumab in patients with metastatic colorectal cancer. Onkologie. 2003;26:44-48.

19. Wang LC, Lo A, Scholler J, et al. Targeting fibroblast activation protein in tumor stroma with chimeric antigen receptor $\mathrm{T}$ cells can inhibit tumor growth and augment host immunity without severe toxicity. Cancer Immunol Res. 2014;2: $154-166$.

20. Lo A, Wang LC, Scholler J, et al. Tumor-promoting desmoplasia is disrupted by depleting FAP-expressing stromal cells. Cancer Res. 2015;75:2800-2810.

21. Ostermann E, Garin-Chesa P, Heider KH, et al. Effective immunoconjugate therapy in cancer models targeting a serine protease of tumor fibroblasts. Clin Cancer Res. 2008;14:4584-4592.

22. Brennen WN, Rosen DM, Wang H, Isaacs JT, Denmeade SR. Targeting carcinoma-associated fibroblasts within the tumor stroma with a fibroblast activation protein-activated prodrug. J Natl Cancer Inst. 2012;104:1320-1334.

23. LeBeau AM, Brennen WN, Aggarwal S, Denmeade SR. Targeting the cancer stroma with a fibroblast activation protein-activated promelittin protoxin. $\mathrm{Mol}$ Cancer Ther. 2009;8:1378-1386.

24. Lee J, Fassnacht M, Nair S, Boczkowski D, Gilboa E. Tumor immunotherapy targeting fibroblast activation protein, a product expressed in tumor-associated fibroblasts. Cancer Res. 2005;65:11156-11163.

25. Teichgräber V, Monasterio C, Chaitanya K, et al. Specific inhibition of fibroblast activation protein (FAP)-alpha prevents tumor progression in vitro. Adv Med Sci. 2015;60:264-272.

26. Loktev A, Lindner T, Mier W, et al. A new method for tumor imaging by targeting cancer associated fibroblasts. J Nucl Med. April 6, 2018 [Epub ahead of print].

27. Fischer E, Chaitanya K, Wuest T, et al. Radioimmunotherapy of fibroblast activation protein positive tumors by rapidly internalizing antibodies. Clin Cancer Res. 2012;18:6208-6218.

28. Jansen K, Heirbaut L, Verkerk R, et al. extended structure-activity relationship and pharmacokinetic investigation of (4-quinolinoyl)glycyl-2-cyanopyrrolidine inhibitors of fibroblast activation protein (FAP). J Med Chem. 2014;57:3053-3074.

29. Meanwell NA. Improving drug candidates by design: a focus on physicochemical properties as a means of improving compound disposition and safety. Chem Res Toxicol. 2011;24:1420-1456.

30. Testa B, Crivori P, Reist M, Carrupt P-A. The influence of lipophilicity on the pharmacokinetic behavior of drugs: concepts and examples. Perspect Drug Discov Des. 2000;19:179-211. 